\title{
Diagnose „idiopathisches Parkinson-Syndrom“ ist schwierig
}

\author{
Rigor, Tremor und Akinese sind die bekannten \\ Kardinalsymptome des Parkinson-Syndroms, führen \\ aber nicht immer zur korrekten Diagnosestellung.
}

Das idiopathische Parkinson-Syndrom (IPS) wird klinisch auf Basis der Kardinalsymptome Rigor, Tremor und Akinese diagnostiziert. Vor allem für „Nicht-Bewegungs-Spezialisten“ ist die korrekte Diagnose gerade im frühen Krankheitsstadium schwierig, erklärte Professor Jörg B. Schulz, Ärztlicher Direktor der Neurologischen Klinik, Universitätsklinikum Aachen. „Die klinische Diagnose eines IPS wird aktuell nur in $75-80 \%$ autoptisch bestätigt", so Schulz. Das spiegeln auch die Ergebnisse einer kliniko-pathologischen Studie wider, die der diagnostischen Sicherheit in unterschiedlichen Erkrankungsphasen des IPS auf den Grund ging [Adler CH et al., Neurology 2014; 83: 406 - 12]. Hier bestätigte sich die erste klinische Diagnose in nur 68\% der Fälle im weiteren Verlauf der Krankheit und in lediglich $77 \%$ der Fälle wurde die während des klinischen Verlaufs gestellte Diagnose neuropathologisch bestätigt. Differenzierte man die Patienten hinsichtlich der Diagnose bei Erstkontakt in solche, die klar auf eine Medikation ansprachen (PDprob) und andere, die entweder nicht angesprochen hatten oder nicht behandelt worden waren (PDposs), dann unterschied sich die diagnostische Vorhersagegenauigkeit noch stärker: Bei den L-Dopa-Respondern bestätigte die post mortem erhobene Diagnose die bei der Erstvorstellung gestellte in $82 \%$ gegenüber $26 \%$ in der PDposs-Gruppe. Dabei lagen die Ärzte mit ihrer Diagnose bei einem Krankheitsverlauf unter fünf Jahren in nur $53 \%$ der Fälle, bei fünf Jahren und mehr dagegen in $88 \%$ der Fälle richtig

Bewegungsstörungen (Jörg B. Schulz), 8. Neurologie-Update-Seminar,

19. - 20.2.2016 in Mainz

\section{Neue Kriterien der Movement Disorder Society sollen bei der IPS-Diagnose helfen}

\author{
Die Sensitivität und Spezifität der klinischen Diag- \\ nose idiopathisches Parkinson-Syndrom (IPS) lässt zu \\ wünschen übrig. Um beides zu verbessern, hat die \\ Movement Disorder Society (MDS) 2015 in einem \\ Expertenverfahren entwickelte neue Diagnose- \\ kriterien veröffentlicht.
}

Die neuen Kriterien sollen die Diagnose auch bei weniger Erfahrung in drei Schritten im Ausschlussverfahren erleichtern, berichtete Professor Jörg B. Schulz, Aachen ( $\bullet$ Abb. 1) [Postuma RB et al. Mov Disord 2015; 30:1591-601]). Im ersten Schritt müssten die Kernkriterien Bradykinese, Ruhetremor und Rigor dokumentiert werden. Dann sind die Ausschlusskriterien zu berücksichtigen - von den sicheren zerebellären Symptomen bis zum normalen präsynaptischen dopaminergen System im DaTScan - und schließlich wird nach Warnhinweisen beziehungsweise „red flags" gefahndet - angefangen bei rasch progredienter Gehbeeinträchtigung mit Rollstuhlpflicht innerhalb von fünf Jahren nach Symptombeginn bis hin zu bilateralem symmetrischen Parkinson-Syndrom, erklärte Schulz. Als supportive Kriterien gelten neben klarem und deutlichem Ansprechen auf eine dopaminerge Therapie, das Vorhandensein L-Dopa-assoziierter Dyskinesien, der Ruhetremor einer Extremität sowie der Riechverlust oder die verminderte sympathische Innervation des Herzens (MIBG Szintigrafie).

Schulz bewertet die operationalisierten Kriterien als Schritt in die richtige Richtung. Werden die Schritte wie empfohlen konsequent „abgearbeitet“, könnten auch Nichtspezialisten ein idiopathisches Parkinson-Syndrom mit hoher Sicherheit identifizieren. Der Ankündigung, unter Beibehaltung der motori-

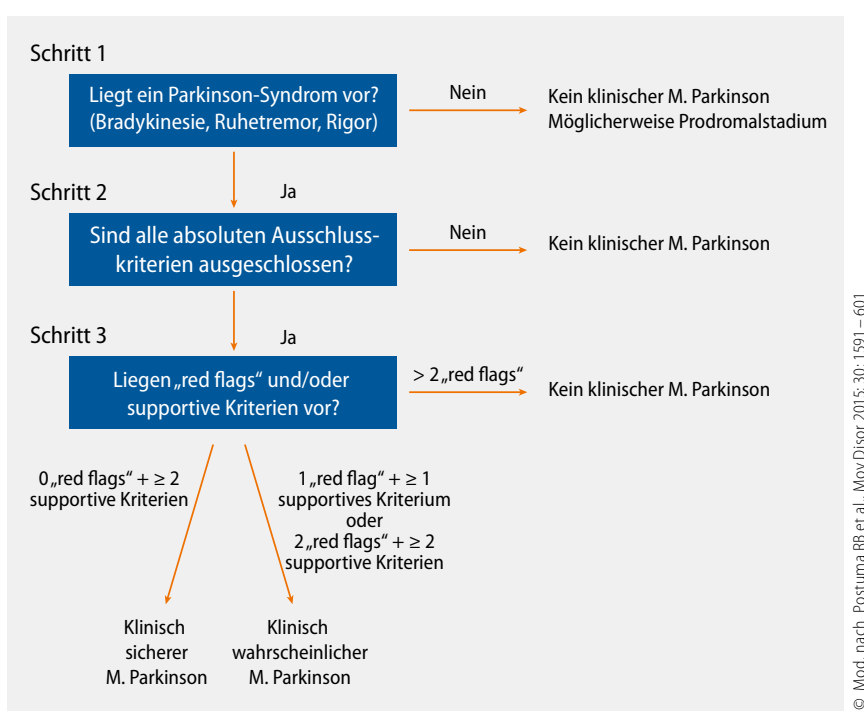

1 Die neuen Diagnosekriterien der Movement Disorder Society

schen Kernkriterien vor allem in frühen Stadien des IPS nicht motorische Kriterien stärker zu gewichten, werde die MDS-Aktualisierung aber nicht ausreichend gerecht, sodass die Sensitivität der Diagnose wohl nicht gebessert wird, diskutierte Schulz.

Ute Ayazpoor, freie Medizinjournalistin

Bewegungsstörungen (Jörg B. Schulz), 8. Neurologie-Update-Seminar, 19. - 20.2.2016 in Mainz 